EESTI NSV TEADUSTE AKADEEMIA TOIMETISED. XIV KÖIDE FOOSIKA-MATEMAATIKA- JA TEHNIKATEADUSTE SEERIA. 1965, NR. 2

ИЗВЕСТИЯ АКАДЕМИИ НАУК ЭСТОНСКОЙ ССР. ТОМ ХІV СЕРИЯ ФИЗИКО-МАТЕМАТИЧЕСКИХ И ТЕХНИЧЕСКИХ НАУК. 1965, № 2

\title{
T. ТОБИАС
}

\section{О НЕКОТОРЫХ ПРИБЛИЖЕННЫХ ФОРМУЛАХ, НАИЛУЧШИХ В СМЫСЛЕ МЕРЫ ВИНЕРА}

\section{Сообщение II}

Настоящая работа является непосредственным продолжением статьи [1], где дана постановка проблемы. Там же приведены все обозначения и определения.

\section{4. Приближение суммами типа Фурье}

Рассмотрим приближенную формулу

$$
y(t) \approx \sum_{k=1}^{n} \varphi_{k}(t) V_{k}(y)
$$

где теперь $V_{k}(y)=\int_{0}^{1} a_{k}(t) y(t) d t$. Систему функций $\left\{a_{k}(t)\right\}$ выберем из условия ортогональности

$$
\begin{aligned}
\left(V_{k}, V_{l}\right) & =\int_{C} \int_{0}^{1} a_{k}(s) T x(s) d s \int_{0}^{1} a_{l}(t) T x(t) d t d_{\mathbb{W}} x= \\
& =\int_{0}^{1} \int_{0}^{1} a_{k}(s) a_{l}(t) R(s, t) d s d t=0,
\end{aligned}
$$

если $k \neq l$.

Тогда легко видеть, что

$$
\begin{aligned}
\varphi_{k}(t) & =\frac{1}{D_{k}} \int_{C} T x(t) \int_{0}^{1} a_{k}(s) T x(s) d s d_{W} x= \\
& =\frac{1}{D_{k}} \int_{0}^{1} a_{k}(s) R(s, t) d s,
\end{aligned}
$$

где

$$
D_{k}=\int_{C}\left[\int_{0}^{1} a_{k}(t) T x(t) d t\right]^{2} d_{\mathbb{W}} x=\int_{0}^{1} \int_{0}^{1} a_{k}(s) a_{k}(t) R(s, t) d s d t
$$


Каждой системе функций $\left\{a_{k}(t)\right\}$, удовлетворяющих условию (4.2), соответствует свое разложение (4.1). Функции $\left\{a_{k}(t)\right\}$ можно выбирать, например, как полиномы или собственные функции симметрического ядра $R(s, t)$. Рассмотрим несколько примеров.

Пусть $x(t) \in C$. Соответствующее интегральное уравнение

$$
a_{k}(t)=\lambda_{k} \int_{0}^{1} \min (s, t) a_{k}(s) d s
$$

эквивалентно краевой задаче

$$
a_{k}^{\prime \prime}(t)=-\lambda_{k} a_{k}(t), \quad a_{k}(0)=a_{k}^{\prime}(1)=0,
$$

решением которой являются

$$
a_{k}(t)=\sqrt{2} \sin \frac{2 k-1}{2} \pi t, \quad \lambda_{k}=\frac{\pi^{2}(2 k-1)^{2}}{4} .
$$

Легко видеть, что

$$
D_{k}=\frac{4}{\pi^{2}(2 k-1)^{2}} \quad \text { и } \quad \varphi_{k}(t)=\sqrt{2} \sin \frac{2 k-1}{2} \pi t .
$$

Искомое разложение

$$
x(t) \approx \sum_{k=1}^{n} \int_{0}^{1} \sqrt{2} \sin \frac{2 k-1}{2} \pi t x(t) d t \sqrt{2} \sin \frac{2 k-1}{2} \pi t
$$

оказалось отрезком ряда Фурье функции $\left.x(t){ }^{[3}\right]$.

Найдем теперь соответствующую систему полиномов $\left\{a_{k}(t)\right\}$. Используя теорему Мерсера и интегрируя по частям, получим в силу равенства Парсеваля

$$
\begin{gathered}
\int_{0}^{1} \int_{0}^{1} a_{k}(t) a_{l}(s) \min (s, t) d s d t= \\
=\int_{0}^{1} \int_{0}^{1} a_{k}(t) a_{l}(s) \sum_{i=1}^{\infty} \frac{\sqrt{2} \sin \frac{2 i-1}{2} \pi s \sqrt{2} \sin \frac{2 i-1}{2} \pi t}{\frac{(2 i-1)^{2} \pi^{2}}{4}} d s d t=
\end{gathered}
$$$$
=\sum_{i=1}^{\infty} \int_{0}^{1} \int_{t}^{1} a_{k}(u) d u \sqrt{2} \cos \frac{2 i-1}{2} \pi t d t \int_{0}^{1} \int_{s}^{1} a_{l}(v) d v \sqrt{2} \cos \frac{2 i-1}{2} \pi s d s=
$$$$
=\int_{0}^{1} \int_{i}^{1} a_{k}(u) d u \int_{t}^{1} a_{l}(v) d v d t
$$

По условию $\int_{t}^{1} a_{k}(u) d u$ является полиномом $Q_{k+1}(t)$ степени $k+1$; при этом $Q_{k+1}(1)=0$ : 


$$
Q_{k+1}(t)=\int_{t}^{1} a_{k}(u) d u=(1-t) P_{k}(t)
$$

Из условий (4.2) и (4.5) видно, что полиномы $P_{k}(t)$ должны быть ортогональными с весом $(1-t)^{2}$, т. е. $P_{k}(t)$ представляют собой полиномы Якоби. Выберем их нормированными:

$$
P_{k}(t)=\frac{(-1)^{k} \sqrt{2 k+3}}{k !(1-t)^{2}} \frac{d^{k}}{d t^{k}}\left[t^{k}(1-t)^{k+2}\right] .
$$

Полиномы $\left\{a_{k}(t)\right\}$ найдем из соотношения (4.6).

Так как

$$
\varphi_{k}(t)=\int_{0}^{1} a_{k}(s) \min (s, t) d s=\int_{0}^{t}(1-s) P_{k}(s) d s,
$$

To $\left[{ }^{3}\right]$

$$
x(t) \approx \sum_{k=0}^{n} \int_{0}^{1} a_{k}(s) x(s) d s \int_{0}^{t}(1-s) P_{k}(s) d s
$$

Пусть теперь $y(t)=x^{2}(t)-t$, и рассмотрим случай полиномов. После простых преобразований получим

$$
\int_{0}^{1} \int_{0}^{1} a_{k}(s) a_{l}(t) 2 \min ^{2}(s, t) d s d t=4 \int_{0}^{1} t \int_{i}^{1} a_{k}(u) d u \int_{t}^{1} a_{l}(v) d v d t,
$$

поэтому полиномы $\left\{a_{k}(t)\right\}$ должны удовлетворять условию

$$
\int_{0}^{1} t \int_{t}^{1} a_{k}(u) d u \int_{t}^{1} a_{l}(v) d v d t=\frac{1}{4} \delta_{k, l}
$$

Обозначая

$$
\int_{t}^{1} a_{k}(u) d u=(1-t) P_{k}(t)
$$

легко найдем

и

$$
\begin{gathered}
P_{k}(t)=\frac{(-1)^{k} \sqrt{(2 k+4)(k+3)}}{k ! 2 \sqrt{k+1} t(1-t)^{2}} \frac{d^{k}}{d t^{k}}\left[(1-t)^{k+2} t^{k+1}\right] \\
\varphi_{k}(t)=4 \int_{0}^{t} s(1-s) P_{k}(s) d s .
\end{gathered}
$$

Искомое разложение:

$$
x^{2}(t)-t \approx 4 \sum_{k=0}^{n} \int_{0}^{1} a_{k}(t)\left[x^{2}(t)-t\right] d t \int_{0}^{t} s(1-s) P_{k}(s) d s .
$$


Рассмотрим случай $z(t)=y(t)-t X$, где $y(t) \in C_{1, x}$. Интегральное уравнение

$$
a_{k}(t)=\lambda_{k} \int_{0}^{1}[\min (s, t)-s t] a_{k}(s) d s
$$

эквивалентно краевой задаче

$$
a_{k}^{\prime \prime}(t)+\lambda_{k} a_{k}(t)=0, \quad a_{k}(0)=a_{k}(1)=0,
$$

решением которой являются $a_{k}(t)=\sqrt{2} \sin k \pi t, \lambda_{k}=k^{2} \pi^{2}$. Очевидно,

и

$$
\varphi_{k}(t)=\sqrt{2} \sin k \pi t
$$

$$
z(t) \approx \sum_{k=1}^{n} \int_{0}^{1} \sqrt{2} \sin k \pi t z(t) d t \sqrt{2} \sin k \pi t
$$

Разложение по полиномам аналогично разложению (4.7).

Если $y(t) \in C^{(m)}$, то нетрудно видеть, что $\left\{a_{k}(t)\right\}$ можно выбирать собственными функциями краевой задачи

$$
\begin{gathered}
a_{k}^{(2 m+2)}(t)+(-1)^{m} \lambda_{k} a_{k}(t)=0, \\
a_{k}(0)=\ldots=a_{k}^{(m)}=0 ; \quad a_{k}^{(m+1)}(1)=\ldots=a_{k}^{(2 m+1)}(1)=0 .
\end{gathered}
$$

В случае $m=1$ ([4], стр. 693)

$$
\begin{gathered}
\lambda_{k}=k^{4}, a_{k}(t)=(\operatorname{ch} k+\cos k)(\operatorname{sh} k t-\sin k t)- \\
-(\operatorname{sh} k+\sin k)(\operatorname{ch} k t-\cos k t),
\end{gathered}
$$

где $k$ является решением уравнения

$$
\cos k \operatorname{ch} k+1=0
$$

\section{5. Формулы дифференцирования}

Как и ранее, рассмотрим формулы, использующие значения функции лишь в двух-трех точках. Вывод более общих формул для пространства $C^{(1)}$ можно найти в работе [ $\left.{ }^{2}\right]$.

Для сравнения выпишем известные формулы:

$$
\begin{aligned}
& y^{\prime}\left(t_{k}\right) \approx \frac{y\left(t_{k}\right)-y\left(t_{k-1}\right)}{t_{k}-t_{k-1}} \\
& y^{\prime}\left(t_{k}\right) \approx \frac{y\left(t_{k+1}\right)-y\left(t_{k-1}\right)}{t_{k+1}-t_{k-1}} \\
& y^{\prime}\left(t_{k}\right) \approx \frac{y\left(t_{k+1}\right)-y\left(t_{k}\right)}{t_{k+1}-t_{k}}
\end{aligned}
$$




$$
y^{\prime \prime}\left(t_{k}\right) \approx \frac{y\left(t_{k+1}\right)-2 y\left(t_{k}\right)+y\left(t_{k-1}\right)}{\left(t_{k+1}-t_{k-1}\right)^{2}}
$$

со среднеквадратичніой ошибкой $\delta^{\prime}$.

Пусть $y(t) \in C^{(1)}$, и рассмотрим приближенную формулу

$$
y^{\prime}\left(t_{k}\right) \approx C_{1} y\left(t_{k-1}\right)+C_{2} y\left(t_{k}\right)
$$

Используем ортогональную систему ([1], (2.5)) :

$$
\begin{gathered}
V_{k-1}=\frac{y\left(t_{k-1}\right)}{t_{k-1}} ; \quad D_{k-1}=\frac{t_{k-1}}{3} ; \\
V_{k}=\frac{y\left(t_{k}\right)-y\left(t_{k-1}\right)}{t_{k}-t_{k-1}}-\frac{y\left(t_{k-1}\right)}{t_{k-1}}-\frac{1}{2} \frac{y\left(t_{k-1}\right)}{t_{k-1}} ; \quad D_{k}=\frac{4 t_{k}-t_{k-1}}{12} .
\end{gathered}
$$

Коэффициенты приближенной формулы

$$
y^{\prime}\left(t_{k}\right) \approx \varphi_{k-1} V_{k-1}+\varphi_{k} V_{k}
$$

легко вычислить:

$$
\begin{gathered}
\varphi_{k-1}=\frac{1}{D_{k-1}} \int_{C} x\left(t_{k}\right) \frac{1}{t_{k-1}} \int_{0}^{t_{k-1}} x(t) d t d_{W} x=\frac{3}{t_{k-1}} \frac{t_{k-1}}{2} ; \\
\varphi_{k}=\frac{12}{4 t_{k}-t_{k-1}}\left(\frac{t_{k}}{2}-\frac{t_{k-1}}{4}\right) .
\end{gathered}
$$

Если $t_{k}=\frac{k}{n}$, то получим

$$
y^{\prime}\left(\frac{k}{n}\right) \approx \frac{3 n}{3 k+1}\left[(k+1) y\left(\frac{k}{n}\right)-\frac{k^{2}}{k-1} y\left(\frac{k-1}{n}\right)\right]
$$

с дисперсией $\delta=\frac{k}{(3 k+1) n}$ (для формулы $\left.(5.1) \delta^{\prime}=\frac{1}{3 n}\right)$. Точно таким же способом получим

$$
y^{\prime}\left(\frac{k}{n}\right) \approx \frac{3 n}{3 k+4}\left[k y\left(\frac{k+1}{n}\right)-\frac{k^{2}-2}{k} y\left(\frac{k}{n}\right)\right],
$$

$\delta=\frac{k}{(3 k+4) n}\left(\right.$ дисперсия формулы (5.3) $\left.\delta^{\prime}=\frac{1}{3 n}\right)$

พ

$$
y^{\prime}\left(\frac{k}{n}\right) \approx \frac{3 n}{2(3 k+5)}\left[(k+2) y\left(\frac{k+1}{n}\right)-\frac{k^{2}+k-1}{k-1} y\left(\frac{k-1}{n}\right)\right]
$$

с дисперсией $\delta=\frac{2 k+3}{4 n(3 k+5)}\left(\right.$ соответствующая $\left.\delta^{\prime}=\frac{1}{6 n}\right)$.

Даем формулы дифференцирования, которые используют значения функции в трех точках. K системе (5.5), (5.6) добавим еще функционал 


$$
\begin{gathered}
V_{k+1}=\frac{y\left(t_{k+1}\right)-y\left(t_{k}\right)}{t_{k+1}-t_{k}}-\frac{y\left(t_{k}\right)-y\left(t_{k-1}\right)}{t_{k}-t_{k-1}}- \\
-2 \frac{t_{k}-t_{k-1}}{4 t_{k}-t_{k-1}}\left[\frac{y\left(t_{k}\right)-y\left(t_{k-1}\right)}{t_{k}-t_{k-1}}-\frac{3}{2} \frac{y\left(t_{k-1}\right)}{t_{k-1}}\right]
\end{gathered}
$$

с дисперсией

$$
D_{k+1}=\frac{t_{k+1}-t_{k-1}}{3}-\frac{1}{3} \frac{\left(t_{k}-t_{k-1}\right)^{2}}{4 t_{k}-\frac{1}{t_{k-1}}} .
$$

Вычислим коэффициенты приближенной формулы

$$
\begin{gathered}
y^{\prime}\left(t_{k}\right) \approx \varphi_{k-1} V_{k-1}+\varphi_{k} V_{k}+\varphi_{k+1} V_{k+1} ; \\
\varphi_{k-1}=\frac{1}{D_{k-1}} \int_{C} x\left(t_{k}\right) V_{k-1} d_{W} x=\frac{3}{t_{k-1}} \frac{t_{k-1}}{2} ; \quad \varphi_{k}=3 \frac{2 t_{k}-t_{k-1}}{4 t_{k}-t_{k-1}} ; \\
\varphi_{k+1}=\frac{1}{D_{k+1}}\left[\frac{t_{k}-t_{k-1}}{2}-\frac{t_{k}-t_{k-1}}{4 t_{k}-t_{k-1}} t_{k}+\frac{t_{k}-t_{k-1}}{4 t_{k}-t_{k-1}} \frac{t_{k-1}}{2}\right] .
\end{gathered}
$$

В случае $t_{k}=\frac{k}{n}$ легко получить:

$$
y^{\prime}\left(\frac{k}{n}\right) \approx \frac{3 n}{6 k+1}\left[-\frac{k^{2}}{k-1} y\left(\frac{k-1}{n}\right)+y\left(\frac{k}{n}\right)+k y\left(\frac{k+1}{n}\right)\right]
$$

и $\delta=\frac{k}{n(6 k+1)}$.

Аналогично:

$$
\begin{gathered}
y^{\prime}\left(\frac{k-1}{n}\right) \approx \frac{n}{2(6 k+1)}\left[\frac{-15 k^{2}+30 k+6}{k-1} y\left(\frac{k-1}{n}\right)+\right. \\
\left.+18(k-1) y\left(\frac{k}{n}\right)-3(k-1) y\left(\frac{k+1}{n}\right)\right], \quad \delta=\frac{7(k-1)}{4 n(6 k+1)}
\end{gathered}
$$

и

$$
\begin{gathered}
y^{\prime}\left(\frac{k+1}{n}\right) \approx \frac{n}{6 k+1}\left[\frac{3 k^{2}}{2(k-1)} y\left(\frac{k-1}{n}\right)-3(3 k+1) y\left(\frac{k}{n}\right)+\frac{3(5 k+1)}{2} y\left(\frac{k+1}{n}\right)\right] \\
\delta=\frac{7 k+1}{4 n(6 k+1)} .
\end{gathered}
$$

Пусть $y(t) \in C^{(2)}$. Рассмотрим приближенную формулу вида (5.7), где примем сразу $t_{k}=\frac{k}{n}$.

$$
\begin{aligned}
V_{k-1} & =y\left(\frac{k-1}{n}\right) ; \quad D_{k-1}=\frac{(k-1)^{5}}{20 n^{5}} ; \quad \varphi_{k-1}=\frac{1}{D_{k-1}} \frac{(k-1)^{3}(3 k+1)}{24 n^{4}} \\
V_{k} & =y\left(\frac{k}{n}\right)-y\left(\frac{k-1}{n}\right) \frac{6 k^{2}+3 k+1}{6(k-1)^{2}} ; \quad D_{k}=\frac{15 k^{3}+15 k^{2}+5 k+1}{720 n^{5}} \\
\varphi_{k} & =\frac{1}{D_{k}} \frac{3 k^{3}+9 k^{2}+5 k+1}{144 n^{4}}
\end{aligned}
$$


Теперь легко найти и окончательную формулу:

$$
\begin{aligned}
& y^{\prime}\left(\frac{k}{n}\right) \approx \frac{5 n}{15 k^{3}+15 k^{2}+5 k+1}\left[\left(3 k^{3}+9 k^{2}+5 k+1\right) y\left(\frac{k}{n}\right)-\frac{3 k^{4}(k+1)}{(k-1)^{2}} y\left(\frac{k-1}{n}\right)\right], \\
& \text { где } \delta=\frac{k^{3}(5 k+4)}{12 n^{3}\left(15 k^{3}+15 k^{2}+5 k+1\right)}\left(\text { дисперсия формулы }(5.1) \delta^{\prime}=\frac{15 k-7}{60 n^{3}}\right) .
\end{aligned}
$$

Аналогично:

$$
\begin{aligned}
& y^{\prime}\left(\frac{k}{n}\right) \approx \frac{5 n}{15 k^{3}+60 k^{2}+80 k+36}\left[3 k^{2}(k+2) y\left(\frac{k+1}{n}\right)-\right. \\
& \left.-\frac{3 k^{4}+6 k^{3}-10 k^{2}-30 k-18}{k} y\left(\frac{k}{n}\right)\right] \\
& \text { с дисперсией } \delta=\frac{k^{3}(5 k+9)}{12 n^{3}\left(15 k^{3}+60 k^{2}+80 k+36\right)} \quad\left(\delta^{\prime}=\frac{15 k+3}{60 n^{3}}\right) \\
& y^{\prime}\left(\frac{k}{n}\right) \approx \frac{5 n}{2\left(15 k^{3}+75 k^{2}+125 k+73\right)}\left[\left(3 k^{3}+15 k^{2}+25 k+8\right) y\left(\frac{k+1}{n}\right)-\right. \\
& \left.-\frac{3 k^{5}+9 k^{4}-2 k^{3}-27 k^{2}-24 k-3}{(k-1)^{2}} y\left(\frac{k-1}{n}\right)\right] \\
& \delta=\frac{6 k^{3}+30 k^{2}+50 k+5}{16 n^{3}\left(15 k^{3}+75 k^{2}+125 k+73\right)} \quad\left(\delta^{\prime}=\frac{13}{120 n^{3}}\right) .
\end{aligned}
$$

Пусть $z(t)=y(t)-\frac{t^{2}}{2} X$, где $y(t) \in C_{1, X}^{(1)}$. Даем сперва соответствующие величины в формуле (5.7) при произвольных узлах.

$$
\begin{aligned}
& V_{k-1}=z\left(t_{k-1}\right) ; \quad D_{k-1}=\frac{t_{k-1}^{3}}{12}\left(4-3 t_{k-1}\right) ; \quad \varphi_{k-1}=\frac{1}{D_{k-1}} \frac{t_{k-1}^{2}}{2}\left(1-t_{k}\right) ; \\
& V_{k}=z\left(t_{k}\right)-z\left(t_{k-1}\right) \frac{6 t_{k}-2 t_{k-1}-3 t_{k}^{2}}{t_{k-1}\left(4-3 t_{k-1}\right)} ; \\
& D_{k}=\frac{t_{k}^{3}}{12}\left(4-3 t_{k}\right)-\frac{t_{k-1}}{12\left(4-3 t_{k-1}\right)}\left(6 t_{k}-2 t_{k-1}-3 t_{k}^{2}\right)^{2} ; \\
& \varphi_{k}=\frac{1}{D_{k}}\left[\frac{t_{k}^{2}}{2}-\frac{t_{k}^{3}}{3}-\frac{t_{k-1}\left(1-t_{k}\right)}{2\left(4-3 t_{k-1}\right)}\left(6 t_{k}-2 t_{k-1}-3 t_{k}^{2}\right)\right] . \\
& \text { В случае } t_{k}=\frac{k}{n} \text { получим: }
\end{aligned}
$$

$$
\begin{aligned}
& z^{\prime}\left(\frac{k}{n}\right) \approx \frac{3 n(n-k)}{\left(3 k n+n-3 k^{2}\right)(k-1)}\left[\left(k^{2}-1\right) z\left(\frac{k}{n}\right)-k^{2} z\left(\frac{k-1}{n}\right)\right] \\
& z^{\prime}\left(\frac{k}{n}\right) \approx \frac{1}{\left(3 k n+4 n-3 k^{2}-6 k-3\right)}\left[\frac{3 k n(2 n-2 k-1)}{2} z\left(\frac{k+1}{n}\right)+\right. \\
&+\left(48 n^{3}-36 n^{2}-84 k n^{2}+36 k^{2} n+12 k^{2} n^{2}+42 k^{3} n^{2}-24 k^{2} n^{3}+\right. \\
&\left.\left.+27 k n-18 k^{4} n-9 k^{3} n\right) \times \frac{1}{2 k(4 n-3 k)} z\left(\frac{k}{n}\right)\right]
\end{aligned}
$$

3 ENSV TA Toimetised F-2 65. 
H

$$
\begin{gathered}
z^{\prime}\left(\frac{k}{n}\right) \approx \frac{3 n}{8\left(5 n-6 k+3 k n-3 k^{2}-3\right)}\left[\left(8 n-9 k+4 k n-4 k^{2}-3\right) z\left(\frac{k+1}{n}\right)+\right. \\
+\left(16 n^{2}-32 k n-16 k n^{2}+20 k^{2} n+21 k^{2}-3 k^{3}+3 k-16 k^{2} n^{2}+28 k^{3} n-\right. \\
\left.\left.-12 k^{4}-9\right) \times \frac{1}{(k-1)(4 n-3 k+3)} z\left(\frac{k-1}{n}\right)\right]
\end{gathered}
$$

с соответствующими дисперсиями:

$$
\begin{gathered}
\delta=\frac{k(n-k)}{n\left(3 k n+n-3 k^{2}\right)} \quad\left(\delta^{\prime}=\frac{4 n-3}{12 n^{2}}\right), \\
\delta=\frac{k\left(12 k^{2}-28 k n-12 n+9 k+16 n^{2}\right)}{4 n(4 n-3 k)\left(3 k n+4 n-3 k^{2}-6 k-3\right)} \quad\left(\delta^{\prime}=\frac{4 n-3}{12 n^{2}}\right)
\end{gathered}
$$

и

$$
\delta=\frac{48 n^{2}-56 k^{2} n+32 k n^{2}-72 k n+24 k^{3}+21 k^{2}-18 k-27}{16 n(4 n-3 k+3)\left(5 n-6 k+3 k n-3 k^{2}-3\right)} \quad\left(\delta^{\prime}=\frac{1}{6 n}\right) .
$$

Рассмотрим, наконец, вычисление второй производной в пространстве $C^{(2)}$. Отметим, что использование лишь двух ординат не дает сходящихся формул.

Прим ер. Наилучшей формулой вида $y^{\prime \prime}\left(t_{k}\right) \approx C_{1} y\left(t_{k-1}\right)+C_{2} y\left(t_{k}\right)$ является $\left(t_{k}=\frac{k}{n}\right)$ :

$$
y^{\prime \prime}\left(\frac{k}{n}\right) \approx \frac{20 n^{2}}{15 k^{3}+15 k^{2}+5 k+1}\left[\left(3 k^{2}+2 k+1\right) y\left(\frac{k}{n}\right)-\frac{k^{3}(k+1)}{(k-1)^{2}} y\left(\frac{k-1}{n}\right)\right]
$$

с дисперсией $\delta=\frac{k\left(5 k^{3}+25 k^{2}+15 k+3\right)}{3 n\left(15 k^{3}+15 k^{2}+5 k+1\right)} . \quad$ Если $k=n$, то $\delta-\frac{1}{9}$.

Используя ортогональную систему

$$
\begin{array}{rc}
V_{k-1}=y\left(\frac{k-1}{n}\right) ; & D_{k-1}=\frac{1}{20} \frac{(k-1)^{5}}{n^{5}} ; \\
V_{k}=y\left(\frac{k}{n}\right)-y\left(\frac{k-1}{n}\right) \frac{6 k^{2}+3 k+1}{6(k-1)^{2}} ; & D_{k}=\frac{15 k^{3}+15 k^{2}+5 k+1}{720 n^{5}} ; \\
V_{k+1}=y\left(\frac{k+1}{n}\right)-\frac{3 k^{2}+9 k+8}{3(k-1)^{2}} y\left(\frac{k}{n}\right)-\frac{2\left(15 k^{3}+45 k^{2}+25 k+8\right)}{15 k^{3}+15 k^{2}+5 k+1} V_{k} \\
D_{k+1}=\frac{100 k^{4}+290 k^{3}+150 k^{2}+30 k+3}{60 n^{5}\left(15 k^{3}+15 k^{2}+5 k+1\right)},
\end{array}
$$

получим:

$$
\begin{gathered}
y^{\prime \prime}\left(\frac{k}{n}\right) \approx \frac{5 n^{2}}{3\left(100 k^{4}+290 k^{3}+150 k^{2}+30 k+3\right)} \times \\
\times\left[\frac{k^{3}\left(900 k^{6}+2250 k^{5}+30 k^{4}-1650 k^{3}-990 k^{2}-288 k-36\right)}{(k-1)^{2}\left(15 k^{3}+15 k^{2}+5 k+1\right)} y\left(\frac{k-1}{n}\right)-\right. \\
-\frac{2\left(900 k^{7}+4050 k^{6}+6150 k^{5}+4080 k^{4}+1110 k^{3}-72 k-18\right)}{15 k^{3}+15 k^{2}+5 k+1} y\left(\frac{k}{n}\right)+ \\
\left.+\left(60 k^{4}+210 k^{3}+108 k^{2}+18 k\right) y\left(\frac{k+1}{n}\right)\right]
\end{gathered}
$$


Дисперсия полученной формулы:

$$
\delta=\frac{16200 k^{7}+56700 k^{6}+70200 k^{5}+43740 k^{4}+15660 k^{3}+3240 k^{2}+324 k}{108 n\left(15 k^{3}+15 k^{2}+5 k+1\right)\left(100 k^{4}+290 k^{3}+150 k^{2}+30 k+3\right)},
$$

в то же время формула (5.4) имеет дисперсию $\delta^{\prime}=\frac{1}{10 n}$.

\section{6. Формула Тэйлора}

Рассмотрим приближенную формулу

$$
y(t) \approx C_{0} y\left(t_{0}\right)+C_{1} y^{\prime}\left(t_{0}\right)+\ldots+C_{m} y^{(m)}\left(t_{0}\right),
$$

где $y(t) \in C^{(m)}$ и $t_{0}<t$.

Наилучшие коэффициенты $C_{k}$, минимизирующие выражение

$$
\int_{C}\left[y(t)-\sum_{k=0}^{m} C_{k} y^{(k)}\left(t_{0}\right)\right]^{2} d_{W} x,
$$

можно определить из системы

$$
\begin{aligned}
& \sum_{k=0}^{m} \frac{1}{(m-k) !(m-i) !} \int_{0}^{t_{0}}\left(t_{0}-u\right)^{m-k}\left(t_{0}-u\right)^{m-i} d u \quad C_{k}= \\
= & \frac{1}{m !(m-i) !} \int_{0}^{t_{0}}(t-u)^{m}\left(t_{0}-u\right)^{m-i} d u \quad(i=0, \ldots, m) .
\end{aligned}
$$

Так как

$$
\begin{aligned}
\sum_{k=0}^{m} \frac{1}{(m-k) !}\left(t_{0}-u\right)^{m-k} \frac{\left(t-t_{0}\right)^{k}}{k !} & =\sum_{k=0}^{m} \frac{1}{m !} C_{m}^{k}\left(t_{0}-u\right)^{m-k}\left(t-t_{0}\right)^{k}= \\
& =\frac{1}{m !}(t-u)^{m},
\end{aligned}
$$

то решением системы (6.2) являются $C_{k}=\frac{\left(t-t_{0}\right)^{k}}{k !}$, т. е. наилучшей формулой вида (6.1) в пространстве $C^{(m)}$ является формула Тэйлора.

Для пространства $C^{(1)}$ формула $y\left(t_{0}+\varepsilon\right) \approx y\left(t_{0}\right)+y^{\prime}\left(t_{0}\right) \varepsilon$ имеет дисперсию $\delta=\frac{\varepsilon^{3}}{3}$, в пространстве $C^{(2)}$ дисперсией формулы $y\left(t_{0}+\varepsilon\right) \approx$ $\approx y\left(t_{0}\right)+y^{\prime}\left(t_{0}\right) \varepsilon+\frac{y^{\prime \prime}\left(t_{0}\right)}{2} \varepsilon^{2}$ являетсгं $\delta=\frac{1}{20} \varepsilon^{5}$.

Пусть $p<m$ и $y(t) \in C^{(m)}$. В формуле

$$
y(t) \approx C_{0} g\left(t_{0}\right)+\ldots+C_{p} y^{(p)}\left(t_{0}\right)
$$

наилучшие коэффициенты $C_{k}$ уже не являются коэффициентами Тэйлора. 
Рассмотрим формулу

$$
y(t) \approx C_{0} y\left(t_{0}\right)+C_{1} y^{\prime}\left(t_{0}\right)
$$

в пространстве $y(t) \in C^{(2)}$. Нетрудно получить для определения $C_{0}$ и $C_{1}$ систему

$$
\left\{\begin{array}{l}
\frac{t_{0}^{2}}{5} C_{0}+\frac{t_{0}}{2} C_{1}=\frac{1}{3} t^{2}-\frac{1}{6} t_{0} t+\frac{1}{30} t_{0}^{2} \\
\frac{t_{0}^{2}}{4} C_{0}+\frac{2}{3} t_{0} C_{1}=\frac{1}{2} t^{2}-\frac{1}{3} t_{0} t+\frac{1}{12} t_{0}^{2}
\end{array}\right.
$$

откуда $C_{0}=\frac{20 t_{0} t-10 t^{2}-7 t_{0}^{2}}{3 t_{0}^{2}}, \quad C_{1}=\frac{\left(2 t-t_{0}\right)\left(t-t_{0}\right)}{t_{0}}$.

При $t=t_{0}+\varepsilon$ дисперсией формулы

$$
y\left(t_{0}+\varepsilon\right) \approx \frac{3 t_{0}^{2}-10 \varepsilon^{2}}{3 t_{0}^{2}} y\left(t_{0}\right)+\frac{t_{0}+2 \varepsilon}{t_{0}} y^{\prime}\left(t_{0}\right) \varepsilon
$$

является $\delta=\frac{t_{0}}{36} \varepsilon^{4}+\frac{1}{20} \varepsilon^{5}$. Обычная формула $y\left(t_{0}+\varepsilon\right) \approx y\left(t_{0}\right)+y^{\prime}\left(t_{0}\right) \varepsilon$ имеет дисперсию $\delta^{\prime}=\frac{t_{0}}{4} \varepsilon^{4}+\frac{1}{20} \varepsilon^{5}$.

Если $t<t_{0}$, то формула Тэйлора не обладает вышеуказанным экстремальным свойством $\left(E y^{(p)}(s) \cdot y^{(q)}(t)\right.$ - несимметричная функция переменных $s$ и $t$ ). Наилучшие коэффициенты формулы (6.1) можно определить из системы

$$
\begin{aligned}
& \sum_{k=0}^{m} \frac{1}{(m-k) !(m-i) !} \int_{0}^{t_{0}}\left(t_{0}-u\right)^{m-k}\left(t_{0}-u\right)^{m-i} d u C_{k}= \\
& \quad=\frac{1}{m !(m-i) !} \int_{0}^{t}(t-u)^{m}\left(t_{0}-u\right)^{m-i} d u \quad(i=0, \ldots, m) .
\end{aligned}
$$

Если $y(t) \in C^{(1)}$, то

$$
\left\{\begin{array}{l}
\frac{1}{3} t_{0}^{3} C_{0}+\frac{t_{0}^{2}}{2} C_{1}=\frac{1}{2} t_{0} t^{2}-\frac{1}{6} t^{3} \\
\frac{1}{2} t_{0}^{2} C_{0}+t_{0} C_{1}=\frac{t^{2}}{2}
\end{array}\right.
$$

откуда $C_{0}=\frac{6 t^{2}}{t_{0}^{3}}\left(\frac{t_{0}}{2}-\frac{t}{3}\right), \quad C_{1}=\frac{t^{2}}{t_{0}^{2}}\left(t-t_{0}\right)$.

Дисперсии формул

$y\left(t_{0}-\varepsilon\right) \approx \frac{\left(t_{0}-\varepsilon\right)^{2}\left(t_{0}+2 \varepsilon\right)}{t_{0}^{3}} y\left(t_{0}\right)-\frac{\left(t_{0}-\varepsilon\right)^{2}}{t_{0}^{2}} y^{\prime}\left(t_{0}\right) \varepsilon \quad$ и

$y\left(t_{0}-\varepsilon\right) \approx y\left(t_{0}\right)-y^{\prime}\left(t_{0}\right) \varepsilon$ соответственно $\delta=\frac{\varepsilon^{3}}{3} \frac{\left(t_{0}-\varepsilon\right)^{3}}{t_{0}^{3}}$ и $\delta^{\prime}=\frac{\varepsilon^{3}}{3}$. 
При $y(t) \in C^{(2)}$ можно аналогично вычислить наилучшие коэффициенты формулы $y(t) \approx C_{0} y\left(t_{0}\right)+C_{1} y^{\prime}\left(t_{0}\right)+C_{2} y^{\prime \prime}\left(t_{0}\right)$.

После несложных вычислений получим:

$$
C_{0}=\frac{t^{3}\left(10 t_{0}^{2}-15 t_{0} t+6 t^{2}\right)}{t_{0}^{5}}, \quad C_{1}=\frac{t^{3}\left(3 t-4 t_{0}\right)\left(t_{0}-t\right)}{t_{0}^{4}}, \quad C_{2}=\frac{t^{3}\left(t_{0}-t\right)^{2}}{2 t_{0}^{3}}
$$

Дисперсия полученной формулы

$$
\begin{gathered}
y\left(t_{0}-\varepsilon\right) \approx \frac{\left(t_{0}-\varepsilon\right)^{3}\left(t_{0}^{2}+3 t_{0} \varepsilon+6 \varepsilon^{2}\right)}{t_{0}^{5}} y\left(t_{0}\right)-\frac{\left(t_{0}-\varepsilon\right)^{3}\left(t_{0}+3 \varepsilon\right) \varepsilon}{t_{0}^{4}} y^{\prime}\left(t_{0}\right)+ \\
+\frac{\left(t_{0}-\varepsilon\right)^{3} \varepsilon^{2}}{2 t_{0}^{3}} y^{\prime \prime}\left(t_{0}\right): \quad \delta=\frac{\varepsilon^{5}}{20} \frac{\left(t_{0}-\varepsilon\right)^{5}}{t_{0}^{5}}\left(\text { соответствующая } \delta^{\prime}=\frac{\varepsilon^{5}}{20}\right) .
\end{gathered}
$$

Аналогично:

$$
y(t) \approx \frac{t^{3}\left(20 t_{0}^{2}-25 t_{0} t+8 t^{2}\right)}{3 t_{0}^{5}} y\left(t_{0}\right)+\frac{t^{3}\left(2 t_{0}-t\right)\left(t-t_{0}\right)}{t_{0}^{4}} y^{\prime}\left(t_{0}\right),
$$

или

$$
y\left(t_{0}-\varepsilon\right) \approx \frac{\left(t_{0}-\varepsilon\right)^{3}\left(3 t_{0}^{2}+9 t_{0} \varepsilon+8 \varepsilon^{2}\right)}{3 t_{0}^{5}} y\left(t_{0}\right)-\frac{\left(t_{0}-\varepsilon\right)^{3}\left(t_{0}+\varepsilon\right) \varepsilon}{t_{0}^{4}} y^{\prime}\left(t_{0}\right)
$$

с дисперсией $\delta=\frac{\left(t_{0}-\varepsilon\right)^{5}}{t_{0}^{5}} \frac{\left(5 t_{0} \varepsilon^{4}+4 \varepsilon^{5}\right)}{180}\left(\delta^{\prime}=\frac{t_{0} \varepsilon^{4}}{4}-\frac{7 \varepsilon^{5}}{60}\right)$.

\section{ЛИТЕРАТ У РА}

1. Т о б и а с Т., Изв. АН ЭССР. Сер. физ.-мат. и техн. наук, № 1, 64-78 (1965).

2. С ульдин А. В., Сб. Вероятностные методы и кибернетика, ІІ, Изд. Казанск. ун-та, 1963, стр. $3-35$.

3. С ул ьд и н А. В., Изв. вузов, матем., № 5 (18), 165-179 (1960).

4. К а мке Э., Справочник по обыкновенным дифференциальным уравнениям, М., 1950.

Ннститут кибернетики

Академии наук Эстонской ССР
Поступила в редакцию 26/VI 1964

\section{T. TOBIAS}

\section{MONINGATEST WIENERI MOODU MOTTES PARIMATEST LIGIKAUDSETEST VALEMITEST. II}

Artiklis vaadeldakse lähendamist Fourier' osasummadega, diferentseerimisvalemeid ning Taylori valemit Wieneri ruumis ning teistes mõõduga ruumides. Valemi optimaalsuse kriteeriumiks vōetakse dispersiooni minimaalsus.

\section{T. TOBIAS}

\section{SOME APPROXIMATION FORMULAE WHICH ARE BEST IN THE MEAN OF WIENER'S MEASURE. II}

In this paper we consider the approximation with Fourier's sums, differentation formulae and Taylor's formula on Wiener's and other similar spaces. As a measure for the goodness we use the mean square deviation. 